\title{
Induced Pluripotent Stem Cells at Nanoscale
}

\author{
Dagmar Zeuschner, Karina Mildner, Holm Zaehres, and Hans R. Schöler
}

Reprogramming of mouse and human somatic cells into induced pluripotent stem (iPS) cells has been made possible with the expression of the transcription factor quartet Oct4, Sox2, c-Myc, and Klf4. Here, we compared iPS cells derived from mouse embryonic fibroblasts with the 4 factors to embryonic stem cells by electron microscopy. Both cell types are almost indistinguishable at the ultrastructural level, providing further evidence for the similarity of these 2 pluripotent stem cell populations.

\section{Introduction}

$\mathrm{M}$ OUSE AND HUMAN SOMATIC cells can be genetically reprogrammed into induced pluripotent stem (iPS) cells by the expression of a defined set of factors (Oct4, Sox2, c-Myc, and Klf4, as well as Nanog and LIN28) [1-7]. iPS cells could be generated from mouse and human fibroblasts, as well as from mouse liver, stomach, pancreatic, and neural stem cells, and keratinocytes. The similarity of iPS cells and embryonic stem (ES) cells has been demonstrated in their morphology, global expression profiles, epigenetic status, as well as in vitro and in vivo differentiation potential for both mouse and human cells.

On light microscopy, the morphology of iPS cells is indistinguishable from that of ES cells. In the current study, we compared ES cells to iPS cells derived from mouse embryonic fibroblasts (MEFs) by high-resolution electron microscopy. Both ES and iPS cells were grown under identical conditions. MEFs - the differentiated cells from which the iPS cells had been derived-were also compared with ES cells. Different cellular organelles were examined in detail to assess the overall specialization and differentiation state of the cells.

\section{Materials and Methods}

MEFs were isolated from OG2/Rosa26 (Oct4-GFP) transgenic mice [8] and transduced with retroviral vectors expressing Oct4, Sox2, Klf4, and c-Myc using the MX retroviral vector system as described [1,7]. Oct4-GFP-positive colonies were FACS-sorted and cultured on irradiated MEFs in ESC medium without any further selection. ES cells from the same genetic background and iPS cells were cultivated on mouse embryonic feeder layers according to standard ES cell culture conditions. Cells were initially fixed in 2\% PFA, $2 \%$ GA in $0.1 \mathrm{M}$ cacodylate buffer, $\mathrm{pH} 7.4$, and the samples were further processed either for scanning electron microscopy (SEM) or transmission electron microscopy (TEM). For SEM, the samples were dehydrated stepwise in ethanol and stabilized with carbon-platin before scanning (performed together with nano-Analytics, Münster, Germany). Representative photographs were taken on a Leo-SEM (Zeiss). For TEM, the coverslips were post-fixed in $1 \% \mathrm{OsO}_{4}$ with $1.5 \%$ potassium cyanoferrate in $0.1 \mathrm{M}$ cacodylate buffer and embedded flat in epon. On different elevation level of the culture, 70-nm ultrathin sections of the sample were cut (Leica-UC6 ultramicrotome, Vienna, Austria) and subsequently counterstained with uranyl acetate and lead. The sample was analyzed at $80 \mathrm{kV}$ on a FEI-Tecnai 12 electron microscope (FEI, Eindhoven, Netherlands). In a distance of $0.5 \mu \mathrm{m}$ from the substrate, fibroblasts and iPS/ES cells could be examined in the same section. Up to $50 \mu \mathrm{m}$ only the round iPS and ES colonies remain in the sample. Photographs of selected areas were documented with imaging plates (Ditabis, Pforzheim, Germany). For statistical analysis mitochondria profiles were tagged on randomly taken pictures of cells imaged by a CCD camera (Megaview, Olympus-SIS, Münster, Germany). Minimal and maximal diameter of individual mitochondria was measured in the graphic mode of the camera software (iTEM, Olympus-SIS, Münster, Germany). The arithmetic mean of the values and the standard deviation was calculated accordingly and listed in a table.

\section{Results}

For this study, we reprogrammed MEFs derived from mice carrying the Oct4-GFP transgene [8] into iPS cells by

Department of Cell and Developmental Biology, Max Planck Institute for Molecular Biomedicine, Münster, Germany. 
introducing the 4 factors Oct 4 , Sox2, c-Myc, and Klf4 and by isolating and culturing Oct4-GFP-positive $(+)$ colonies [1,7]. To evaluate potential differences between ES cells and iPS cells at the ultrastructural level in an unbiased way, analysis was performed by investigators blinded to the identity of the samples. As differences in cellular morphology might be caused by different culture conditions, only cells grown in parallel under identical conditions were compared. Examining imaged cells on a SEM (Fig. 1A), the most obvious and apparent difference between iPS cells and fibroblasts can be seen in co-culture: fibroblasts spread spindle-like as single cells flat on the surface of the culture dish, while iPS cells grow in close association as colonies on top of the substrate. It is this latter aspect that is especially reminiscent of an ES cell colony (Fig. 1B). On ultrathin sections (Fig. 1C), iPS cells can be easily distinguished from fibroblasts, as they appear smaller-sized (around $15 \mu \mathrm{m}$ ), mostly regularly shaped and well-aligned. These characteristics can be observed by serial sectioning throughout the entire colony, from the bottom $(0.5 \mu \mathrm{m})$ to the top (about $50 \mu \mathrm{m})$. In serial sections, iPS cell
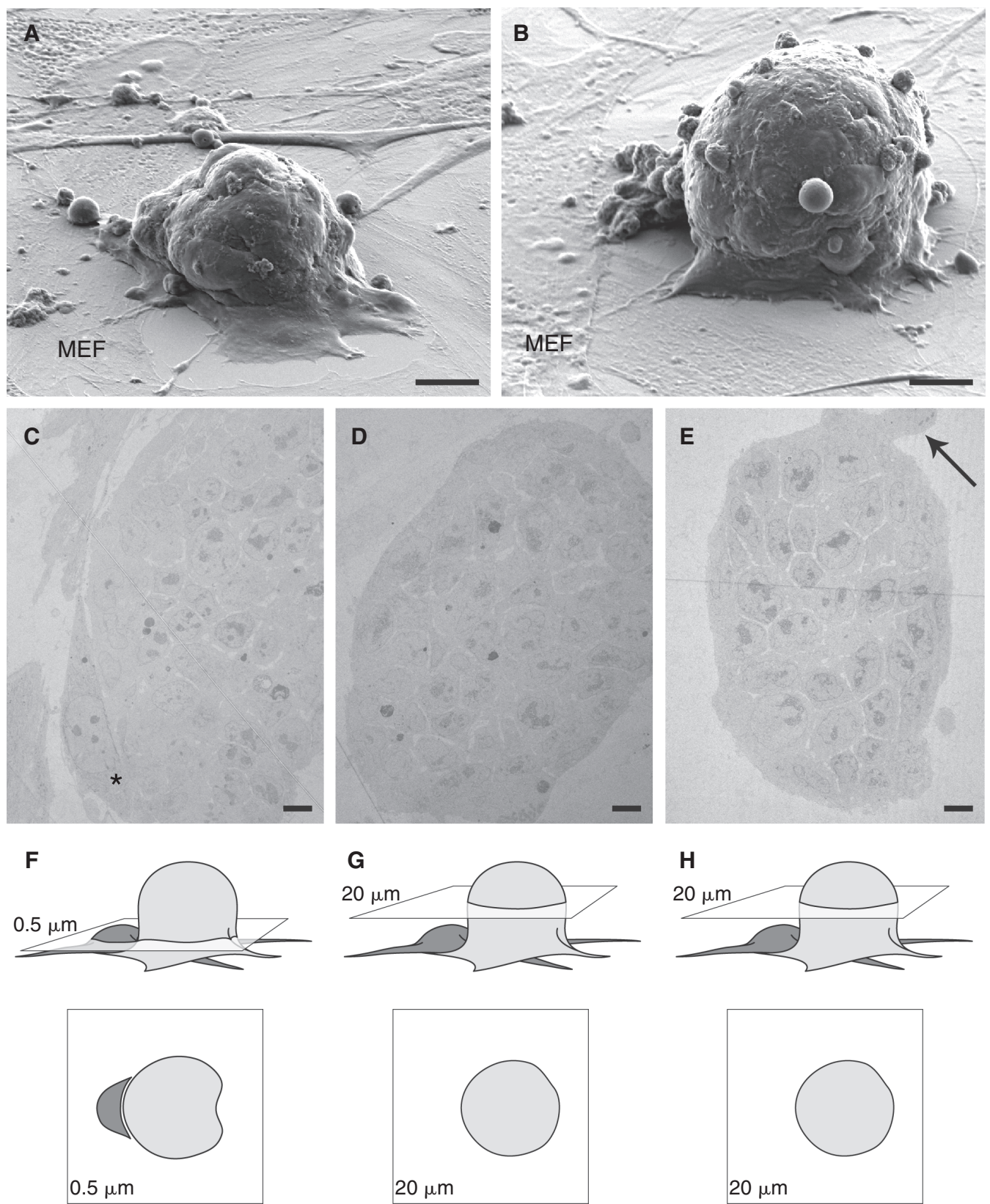

FIG. 1. Induced pluripotent stem (iPS) and embryonic stem (ES) cells grow in spherical colonies, different from fibroblasts. Scanning electron microscopic pictures of iPS (A) and ES (B) cells grown in elevated colonies on a flat mouse embryonic fibroblast (MEF) monolayer. (C) shows the transmission electron microscopic image of an iPS colony, sectioned at a distance of $0.5 \mu \mathrm{m}$ from the bottom of the dish and growing in close association to MEF (asterisks). (D) and (E) are colonies of iPS and ES, respectively, cross-sectioned at a distance of $20 \mu \mathrm{m}$ away from the bottom of the dish. Arrow in (E) points to appended round cell in mitosis. $(\mathbf{F}-\mathbf{H})$ illustrate schematically the section region from $(\mathbf{C})$ to $(\mathbf{D})$ in $3 \mathrm{D}$ and the corresponding profile of the area. Scale bar: $10 \mu \mathrm{m}$. 
colonies are strongly reminiscent of ES cell colonies (Fig. 1E). The outer cell layer of an iPS cell colony is characteristically flattened and neatly organized, as cells make tight contact with their neighbors, and membrane protrusions seal the colony on the outside (Fig. 2D). The contact zone to fibroblasts is rather loose (Fig. 2A). As migrating cells, fibroblasts typically grow in close adhesion to the substrate to which they anchor by focal adhesion (not shown).

Interestingly, iPS and ES cells located in the inner part of the colony maintain wide extracellular spaces, with loose contact points, allowing the constant exchange of nutrients and signaling molecules. In addition, the early endocytic compartment is organized in a notably different way for fibroblasts, iPS, and ES cells (as seen in Supplementary Fig. 1; Supplementary materials are available online at http:// www.liebertpub.com/). Fibroblasts mainly exhibit caveolae, whereas the pluripotent cells show clathrin-mediated endocytosis. A comparison of the nuclei (Fig. 3A-C) emphasizes the extreme difference in cell size. In fibroblasts, arrested by radiation, the cells exhibited typical interphase nuclei, enriched in euchromatin, whereas iPS and ES cells showed regularly condensed chromatin, with frequent mitotic events observed ( 1 out of 50 cell profiles) [9]. We next investigated the nature of mitochondria, a good indicator of the physiological cell state. The most obvious difference in our study was the outline of the mitochondria. In fibroblasts, mitochondria appear mainly tubular-shaped and branched and they display well-developed christae, while in iPS/ES cells, they appear mostly globular shape with only small christae (representative photographs in Fig. 3D-F). To confirm the observed appearance of these organelles, the minimal and maximal axes of randomly selected mitochondria were counted (values in Table 1). In fibroblasts, the mean value for the minimal axis is almost one-fifth less than that for the maximal axis (284 nm, $1352 \mathrm{~nm}$ ), suggesting that tubules are the predominant type of mitochondria. The value of the maximal axis has a high standard deviation indicative of variability in length. In contrast, the mitochondria of iPS
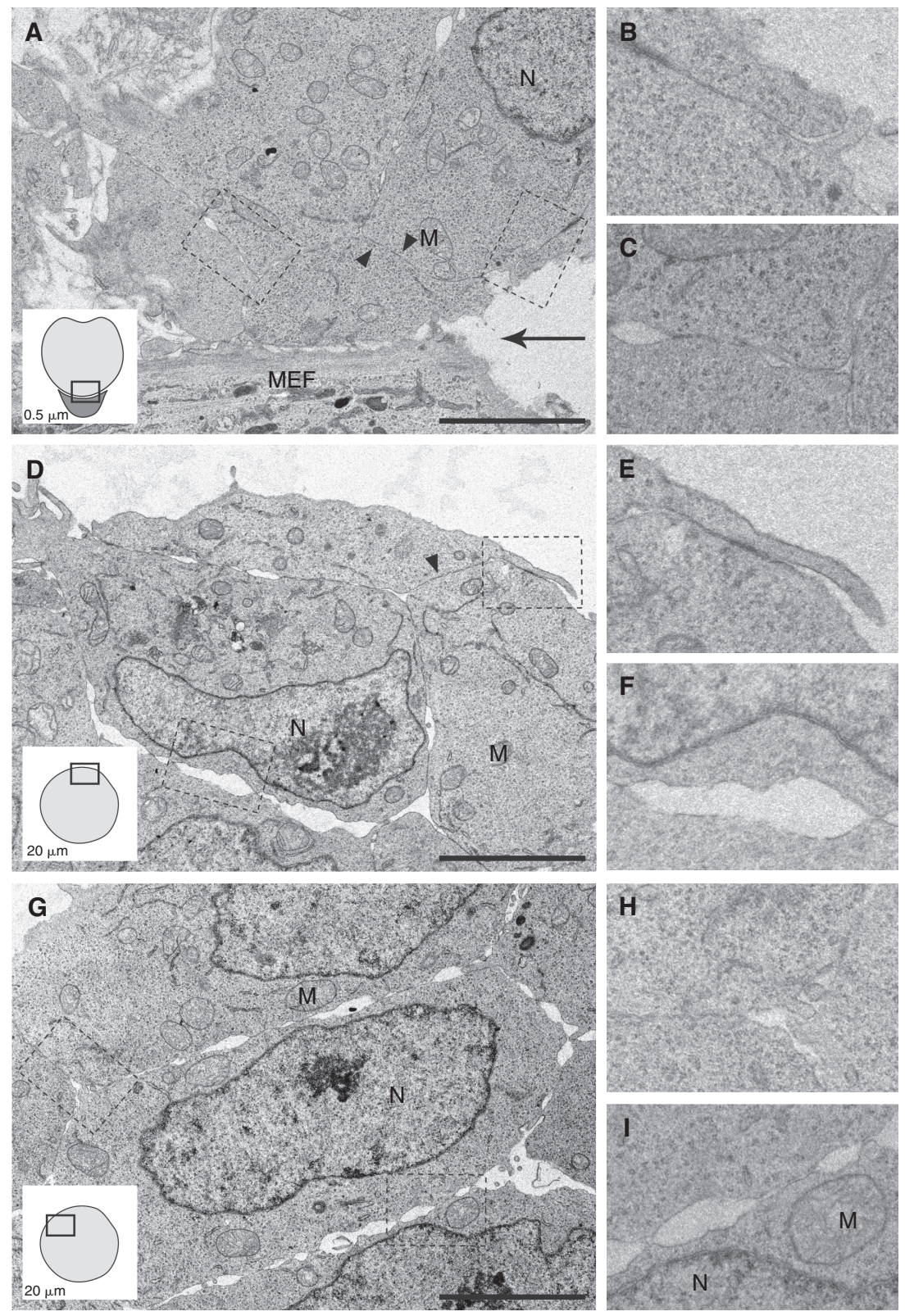

FIG. 2. Cell-cell contacts are established on different levels. (A) exemplifies a relative loose contact zone (arrow) between an embryonic stem (ES) cell colony and a mouse embryonic fibroblast. First cell layer of the ES cell colony is tightly sealed by cell-cell contacts (arrow heads) and pseudopodia-like protrusions (outlined in (B). The inner cells have more punctuated contacts (outlined in (C)). (D) shows cells in induced pluripotent stem (iPS) cell colonies sectioned at $20 \mu \mathrm{m}$, as illustrated in the scheme. Cells make contact with neighboring cells via close contact sites (arrow heads) and pseudopodia (insert (E)). The inner cell layers keep contact mainly via punctuate contact sites (insert (F)). (G) demonstrates an ES cell colony at a distance of $20 \mu \mathrm{m}$ away from the substrate, with outlined sealed contacts in $(\mathbf{H})$ and more loosened contacts sites in (I). The section area is schematically illustrated in the inserts. Abbreviations: N: nucleus. M: mitochondria. Scale bar: $5 \mu \mathrm{m}$. 

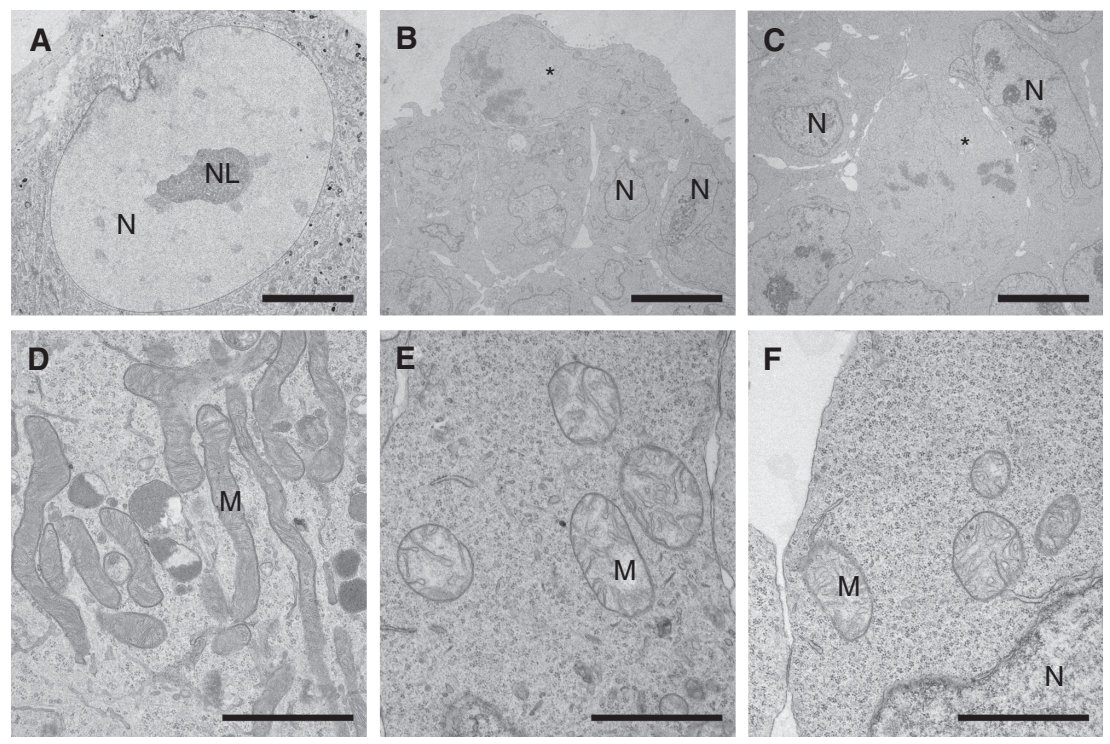

FIG. 3. Evaluation of cellular organelles: nuclear architecture and the morphological appearance of mitochondria. (A) shows the nucleus $(\mathrm{N})$ of a fibroblast, with mainly euchromatin and 1 visible nucleolus (NL). Nuclei of induced pluripotent stem (iPS) cells and embryonic stem (ES) cells are shown in (B) and (C). In iPS and ES cells, mitotic events can be observed frequently (asterisks). Scale bar: $10 \mu \mathrm{m}$ (A-C). In (D), typical mitochondria (M) of fibroblasts are illustrated: they are mostly of tubular shape and enriched in inner membranes (christae). The mitochondria of iPS cells (E) are mostly globular-shaped and possess only few inner membranes. The mitochondria of ES cells are depicted in (F). Scale bar: $1 \mu \mathrm{m}(\mathbf{D}-\mathbf{F})$. and ES cells showed almost similar results for minimal and maximal axes, demonstrating that they mostly appear as a globular shape.

The cytoplasm of fibroblasts is densely packed with membrane-bound organelles, endosomes, lysosomes, with the biosynthetic compartment found in close vicinity (Fig. $4 \mathrm{~A}$ and D). In contrast, the organelles of iPS and ES cells are widely distributed throughout the cytoplasm, containing an abundance of free ribosomes (Fig. 4B, C, E, and F). All the compartments of the early endocytic compartment could be evenly seen in all cells types (Fig. 4A-C). The endoplasmic reticulum (ER) in iPS and ES cells is relatively poorly developed, as only narrow ER elements can be seen in contrast to the wide, complex cisternae in fibroblasts. The Golgi stack is found in a stage that is relatively similar to the ER, supporting the notion that compartment complexity depends on the cargo load, which is rather high for fibroblasts, which secrete components of the extracellular matrix [10].

\section{Discussion}

The morphological appearance of a specific cell type is affected by the cell's physiological milieu and functional needs. Cells organized in tissues show rather small variation

Table 1. Mitochondrial Morphometry

\begin{tabular}{lccc}
\hline $\begin{array}{l}\text { Mitochondria } \\
\text { diameter }(\mathrm{nm})\end{array}$ & $\begin{array}{c}\text { Mouse } \\
\text { embryonic } \\
\text { fibroblast }\end{array}$ & $\begin{array}{c}\text { Induced } \\
\text { pluripotent } \\
\text { stem cells }\end{array}$ & $\begin{array}{c}\text { Embryonic } \\
\text { stem cells }\end{array}$ \\
\hline Minimal axis & $284 \pm 67$ & $616 \pm 165$ & $571 \pm 145$ \\
Maximal axis & $1352 \pm 898$ & $873 \pm 255$ & $816 \pm 258$ \\
\hline
\end{tabular}

$n=60$.

Data are expressed as Mean \pm SD.

Mean value on measurements of minimal and maximal diameter on randomly selected mitochondria. in cell and organelle shape and size, which are balanced dynamically [11]. The genetic reprogramming of a fibroblast to a pluripotent cell leads to changes in the entire cell. Thus, we have selected to examine morphologically distinct cellular structures to assess whether an induced pluripotent cell resembles an embryonic cell or whether it still maintains features of a fibroblast.

We could show that the major cellular organelles of iPS cells adapt to the nature of ES cells and, consequently, iPS cells might also function like ES cells. Cell size and fate are similar, and even complex systems like the early endocytic compartment appear equivalent between iPS and ES cells. In addition, the uptake of nutrients during endocytosis leads to the recruitment of signaling molecules, with different signaling pathways using different endocytic structures. iPS and ES cells mainly exhibit clathrin-mediated endocytosis and will respond differently than fibroblasts, which mainly use caveolae [12,13]. The observed differences in mitochondrial diameter in MEFs and iPS/ES cells can be explained by dynamic adaptation to changes in the cell's energy state, cell cycle status, and developmental stage [14,15].

In summary, the genetic reprogramming of MEFs into iPS cells is accompanied by morphological changes that render iPS cells virtually indistinguishable from ES cells, even at the ultrastructural level. Our study is the first to demonstrate that ES cells and iPS cells are basically indistinguishable at the nanoscale, further substantiating the similarity of these 2 pluripotent stem cell populations, and meeting the current pluripotency criteria [1-7]. The genetic and morphological similarity of iPS and ES cells underscores the considerable potential of both cell types for the in vitro differentiation into cells that could be used for disease modeling and/or as tissue replacement therapies.

\section{Acknowledgment}

We would like to thank Jeanine Mueller-Keuker for providing the schematic illustrations and critical reading the manuscript. 

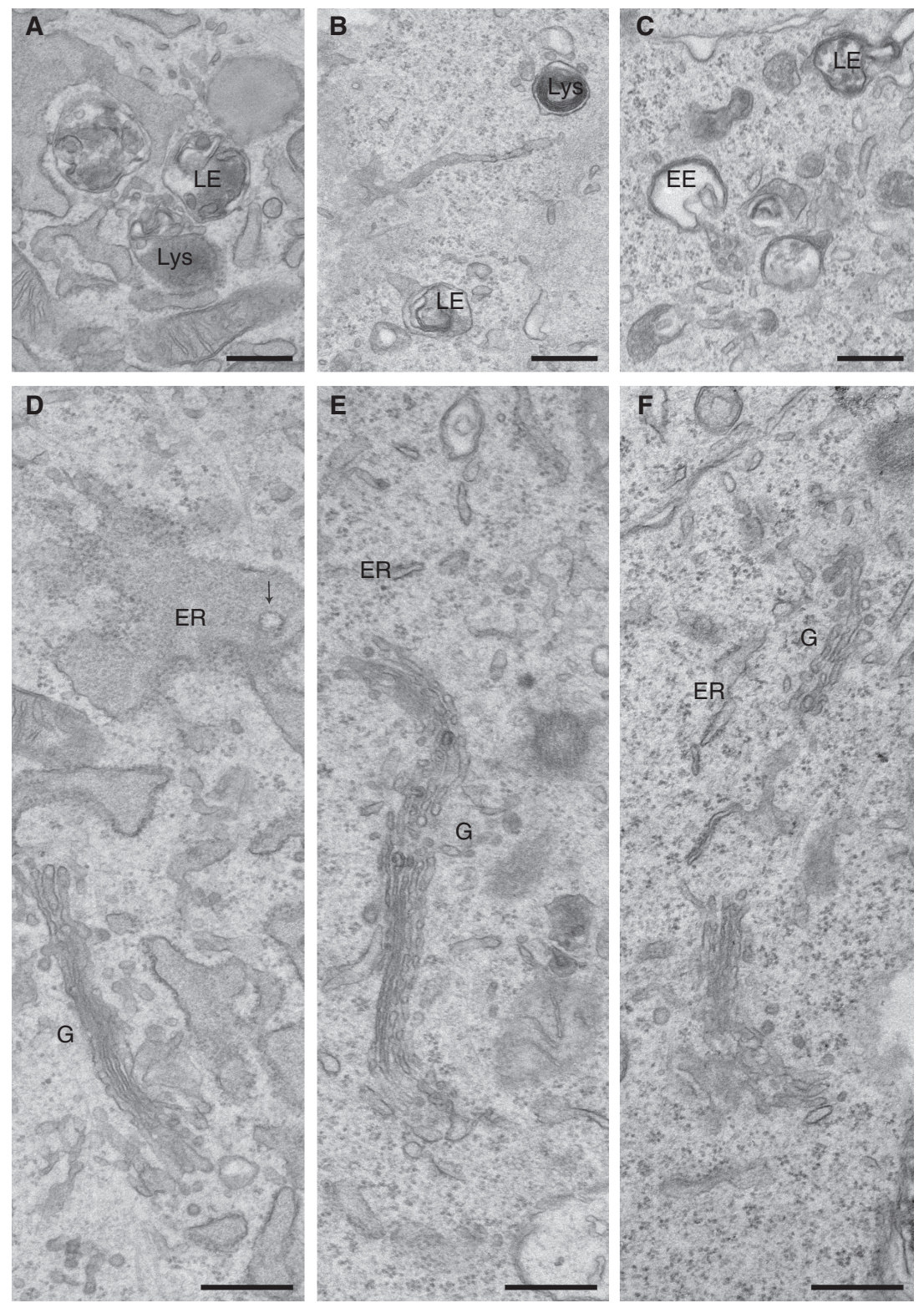

FIG.4. Membranetraffic.(A-C)show representative appearance of the endosomal/ lysosomal membrane compartments in fibroblasts (A), induced pluripotent stem (iPS) cells (B), and embryonic stem (ES) cells (C). Membrane composition of the biosynthetic pathway is demonstrated in panels (D-F), respectively, for fibroblasts, iPS cells, and ES cells. Note the dilated ER and the high fenestration in (D) (arrow). Abbreviations: ER, endoplasmic reticulum; G, Golgi; EE, early endosome; LE, late endosome; Lys, lysosome. Scale bar: $500 \mathrm{~nm}$.

\section{Author Disclosure Statement}

The authors declare no competing financial interests.

\section{References}

1. Takahashi K and S Yamanaka. (2006). Induction of pluripotent stem cells from mouse embryonic and adult fibroblast cultures by defined factors. Cell 126:663-676.

2. Okita K, T Ichisaka and S Yamanaka. (2007). Generation of germline-competent induced pluripotent stem cells. Nature 448:313-317.

3. Wernig $\mathrm{M}$, A Meissner, $\mathrm{R}$ Foreman, $\mathrm{T}$ Brambrink, $\mathrm{M} \mathrm{Ku}, \mathrm{K}$ Hochedlinger, BE Bernstein and R Jaenisch. (2007). In vitro reprogramming of fibroblasts into a pluripotent ES-cell-like state. Nature 448:318-324.

4. Takahashi K, K Tanabe, M Ohnuki, M Narita, T Ichisaka, K Tomoda and S Yamanaka. (2007). Induction of pluripotent stem cells from adult human fibroblasts by defined factors. Cell 131:861-872.

5. Yu J, MA Vodyanik, K Smuga-Otto, J Antosiewicz-Bourget, JL Frane, S Tian, J Nie, GA Jonsdottir, V Ruotti, R Stewart, Slukvin,
II and JA Thomson. (2007). Induced pluripotent stem cell lines derived from human somatic cells. Science 318:1917-1920.

6. Park IH, R Zhao, JA West, A Yabuuchi, H Huo, TA Ince, PH Lerou, MW Lensch and GQ Daley. (2008). Reprogramming of human somatic cells to pluripotency with defined factors. Nature 451:141-146.

7. Kim JB, H Zaehres, G Wu, L Gentile, K Ko, V Sebastiano, MJ Arauzo-Bravo, D Ruau, DW Han, M Zenke and HR Scholer. (2008). Pluripotent stem cells induced from adult neural stem cells by reprogramming with two factors. Nature 454:646-650.

8. Szabo PE, K Hubner, H Scholer and JR Mann. (2002). Allelespecific expression of imprinted genes in mouse migratory primordial germ cells. Mech Dev 115:157-160.

9. Dehghani H, G Dellaire and DP Bazett-Jones. (2005). Organization of chromatin in the interphase mammalian cell. Micron 36:95-108.

10. Wiseman RL, A Koulov, E Powers, JW Kelly and WE Balch. (2007). Protein energetics in maturation of the early secretory pathway. Curr Opin Cell Biol 19:359-367.

11. Rafelski SM and WF Marshall. (2008). Building the cell: design principles of cellular architecture. Nat Rev Mol Cell Biol 9:593-602. 
12. Polo $S$ and PP Di Fiore. (2006). Endocytosis conducts the cell signaling orchestra. Cell 124:897-900.

13. Riemann D, GH Hansen, L Niels-Christiansen, E Thorsen, L Immerdal, AN Santos, A Kehlen, J Langner and EM Danielsen. (2001). Caveolae/lipid rafts in fibroblast-like synoviocytes: ectopeptidase-rich membrane microdomains. Biochem J 354: $47-55$.

14. Chan DC. (2006). Mitochondria: dynamic organelles in disease, aging, and development. Cell 125:1241-1252.

15. Jourdain I, Y Gachet and JS Hyams. (2009). The dynamin related protein Dnm1 fragments mitochondria in a microtubuledependent manner during the fission yeast cell cycle. Cell Motil Cytoskeleton 66:509-523.
Address correspondence to:

Hans R. Schöler

Department of Cell and Developmental Biology Max Planck Institute for Molecular Biomedicine

Röntgenstrasse 20

Münster, NRW 48149

Germany

E-mail: office@mpi-muenster.mpg.de

Received for publication May 7, 2009

Accepted after revision June 15, 2009

Prepublished on Liebert Instant Online June 15, 2009 